\title{
The sensor hub for detecting the developmental characteristics in reading in children on a white vs. coloured background/coloured overlays
}

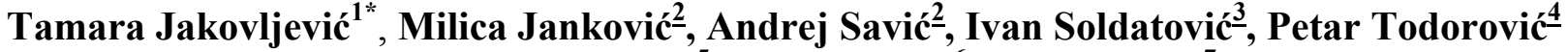 \\ Tadeja Jere Jakulin ${ }^{5}$, Gregor Papa ${ }^{6}$, Vanja Ković ${ }^{7}$ \\ ${ }^{1}$ Sensor technologies, Jožef Stefan International postgraduate school, Ljubljana, Slovenia \\ ${ }^{2}$ University of Belgrade - School of Electrical Engineering, Belgrade, Serbia \\ ${ }^{3}$ Institute of Medical Statistics and Informatics, Faculty of Medicine, University of Belgrade, \\ Belgrade, Serbia \\ ${ }^{4}$ Faculty of Engineering, University of Kragujevac, Kragujevac, Serbia \\ ${ }^{5}$ FTŠ Turistica, UP, Portorož, Slovenia \\ ${ }^{6}$ Jožef Stefan Institute, Ljubljana, Slovenia \\ ${ }^{7}$ Laboratory for Neurocognition and Applied Cognition, Faculty of philosophy, University of \\ Belgrade, Belgrade, Serbia \\ *tamara.jakovljevic@hotmail.com
}

\section{Abstract}

The study investigated the influence of white vs 12 background and overlay colours on the reading process in school age children. Previous research reported that colours could affect reading skills as an important factor of the emotional and physiological state of the body and that reading is one of the most important processes in the maturation of children. The aim of the study was to assess developmental differences between second and third grade students of elementary school and to evaluate differences in electroencephalography (EEG), ocular, electrodermal activities (EDA) and heart rate variability (HRV). In the experiment, the responses of 24 children (12 second and 12 third grade students) to different background and overlay colours were summarized using EEG, eye tracking, EDA and HRV signals. Our findings showed a decreasing trend with age regarding EEG power bands (Alpha, Beta, Delta, Theta) and lower scores of reading duration and eyetracking measures in younger children compared to older children. As shown in the results, HRV parameters showed higher scores in 12 background and overlay colours among second than third grade students which is linearly correlated to the level of stress and readable from EDA measures 
34 as well. The existing study showed the calming effect on second graders in turquoise and blue

35 background colours. Considering other colours separately for each parameter, we assumed that

36 there are no systematic differences in Reading duration, EEG power band, Eye-tracking and EDA

37 measures.

\section{Introduction}

39 Learning to read is a complex process involving both perception and cognition, via integration of

40 visual and auditory information processing and memory, attention and language skills [1].

41 Therefore, reading is a taught skill depending upon a range of perceptual processes and cognitive

42 abilities affecting learning over time and across development [2-4]. One of the key objectives of

43 early education is the learning of reading, and a number of research studies have explored the

44 process of reading skills acquisition in children [2,5-7]. Depending on their individual set of

45 underlying abilities, children will have different developmental profiles of reading skill

46 obtainment, while weaknesses in some abilities may cause reading impairments over time.

47 Individual differences in learning of reading may originate from biological and environmental

48 factors, shaping the development of brain systems involved in the reading process [8]. The current

49 knowledge emphasizes the importance of identifying and treating reading difficulties as early as

50 possible since they may impair academic achievements and increase the risk of social, emotional

51 and mental health problems in children. More specifically, poor reading skills are shown to be

52 associated with increased risks for school dropout, attempted suicide, incarceration, anxiety,

53 depression, and low self-concept [9].

54 There is some limited evidence that colours may impact the reading process, specifically with early

55 school-age children, and those with reading disabilities [10,11]. Going back a few decades

$56[12,13]$, it has been shown that the role of colours in reading dates back to 1958. Jansky [14] 
57 reported the case of a student with a reading deficit who was able to recognize words printed on

58 yellow paper, but unable to recognize words printed on white paper. Previous studies considered

59 the influence of background-, text- or overlay-colour on the actual reading process in children

$60[13,15,16]$. While more recent studies have shown that colours do not influence the reading process

61 and that this could be a placebo [17], others have found that colours may be particularly effective

62 for early readers in school-age children [18].

63 As reading involves sensory integration, attention and memory, those processes may be reflected

64 in the psycho-physiological states of the individual engaged in the reading task. Those states are a

65 result of underlying neural and physiological processes, which are measurable and quantifiable by

66 different biosignal modalities. The goal of this study was to employ multimodal sensor

67 measurements to examine the influence of colour of the content on the reading task in children at

68 different developmental stages. More specifically, we have employed measurements of

69 electroencephalography (EEG), eye-tracking, electrodermal activity (EDA) and heart rate

70 variability (HRV) to assess the influence of background and overlay colour on reading

71 performance in second and third grade students of elementary school. We aimed to address the

72 mechanisms of colour influence on the reading process through electrophysiological correlates of

73 the reader's state, while taking into account the developmental aspect of reading acquisition.

75 This study sheds light on underlying neural, physiological and behavioural processes

76 accompanying the reading task in children. Moreover, this is an initial step towards the possibility

77 of including colour into reading content to improve reading skills in children at different

78 developmental stages.

\section{Method}




\section{Participants}

81 Twenty-five healthy participants were randomly chosen from a single class of the second and third

82 grades of the elementary school "Drinka Pavlović" in Belgrade respectively. The participants ages

83 ranged from 8 to 9 years. Inclusion criteria were that children have no reading and learning

84 disabilities, attention disorders, have normal or corrected to normal vision. Only one child was

85 excluded from the analysis due to the large artefacts in the acquired signals and his data were not

86 used in the statistical analysis.

87 All the subjects underwent the same experimental conditions and participated individually in the

88 small school classroom during the regular time period of the daily school classes. Each child

89 received a short instruction about the experiment setup and design. After the experiment, every

90 child received a small present and diploma for participation in the experiment process.

91 The ethical committee of the Psychology Department of the University of Niš approved the 92 experimental procedure.

93

\section{Experiment setup}

95 During the experiment, each participant was sitting on a chair at a table in the front of a computer

96 monitor and keyboard. At the beginning of the experiment, participants got the instruction to read

97 quietly for themselves the text from the stimuli presentation shown on the computer monitor and

98 to press the space button for the next slide of stimuli presentation. The experiment was repeated in

99 one pseudo randomization of colour background/overlay order starting always with a referent slide

100 (black text on white background). Details about the "experiment" content are explained in the

101 Section Experiment Design. 
102 During the reading process, physiological data were acquired using a sensor hub composed of an 103 eye-tracking system and a portable multimodal EEG/ECG/EDA system, Fig.1. Two laptops were 104 used for data real-time monitoring and storage: one laptop for eye movement monitoring (with

105 additional external computer monitor and keyboard in front of the participant) and another for EEG/ECG/EDA monitoring.

Figure 1. Experiment setup: 1) Eye-tracking system 2) Portable multimodal EEG/ECG/EDA system, 3) Photosensitive sensor for synchronization of multimodal EEG/ECG/EDA system and eye-tracking system.

110 An SMI RED-m 120-Hz portable remote eye tracker (https:/www.smivision. com) was placed

111 below the computer monitor in front of the participant, and it was fixed in place to keep it from

112 accidentally moving. An adjustable chin-rest was used to ensure the same distance from the

113 monitor and table for each participant (a chinrest was $57 \mathrm{~cm}$ away from the eye-tracking sensor

114 and $16 \mathrm{~cm}$ above the table) [19]. The SMI software was used for stimuli presentation (Experiment

115 Centre 3.7) and data collection (iView RED-m).

116 The EEG and ECG signals were recorded using a mobile 24-channel EEG amplifier (SMARTING,

117 mBrainTrain, Belgrade, Serbia) wirelessly communicating with a laptop via Bluetooth. Twenty118 two monopolar EEG channels of Greentek Gelfree-S3 cap (10/20 locations: Fp1, Fp2, F3, F4, C3, $119 \mathrm{C} 4, \mathrm{P} 3, \mathrm{P} 4, \mathrm{O} 1, \mathrm{O} 2, \mathrm{~F} 7, \mathrm{~F} 8, \mathrm{~T} 7, \mathrm{~T} 8, \mathrm{P} 7, \mathrm{P} 8, \mathrm{Fz}, \mathrm{Cz}, \mathrm{Pz}, \mathrm{AFz}, \mathrm{CPz}, \mathrm{POz})$ were recorded. The ground 120 was located at FPz and FCz was used as the reference site. One channel of the amplifier was 121 connected to the surface SKINTACT ECG electrode placed in the left chest region, over the heart, 122 to record ECG signal as a reference for heartbeat detection. EEG and ECG signals were acquired 123 with 24-bit resolution and $250 \mathrm{~Hz}$ sampling rate. The skin-electrode impedances were below the 124 manufacturer recommended value of $1 \mathrm{kOhm}$, prior to the tests. 
125 One channel of the amplifier was used for the synchronization of electrophysiological recordings

126 and eye tracking data. A small photosensitive sensor registering the changes on the screen after

127 each slide was sused with the changes of the black and white screen (200 ms each) and sending a

128 trigger for synchronization of multimodal EEG/ECG/EDA system and eye tracking system, for

129 each event (slide).

130 We used a research prototype for galvanic skin response (electrodermal activity, EDA) recording $131[20]$ that communicates with a laptop via Bluetooth. The sampling rate for EDA data was $40 \mathrm{~Hz}$.

132 SMARTING application has Lab Streaming Layer (LSL) compatibility which enabled 133 synchronization between EDA and EEG/ECG data within a single file of XDF format.

\section{Experiment design}

\section{Stimuli}

136 Participants read a story on the computer monitor. The story was at an adequate level for the

137 second/third-grade of elementary school and selected from the school literature of the Serbian

138 language course. Participants were unfamiliar with the text used in the study.

139 The story "St Sava and the villager without luck" was split into 13 paragraphs: the 1st slide as a

140 referent one - white background with black letters, then 6 slides with black letters on red, blue,

141 yellow, orange, purple and turquoise background and the next 6 slides in the overlay manner which

142 looks like covering black text on a white background with a coloured foil (calculated by the 143 algorithm described in the Section Colour calculation).

144 The experiment started with calibration and validation method would stop on the black slide so

145 the researcher had time to launch multimodal EEG/ECG/EDA system and eye-tracking system for 
146 data acquisition. Next, on the researcher's instruction, the child would press the space button on

147 the keyboard and the first slide with the text appeared on the computer monitor. The participant

148 read text to themselves and then pressed the space button for the next slide to continue the text.

149 After finishing the test, the researcher checked with each child the level of understanding of the 150 story with questions recommended in the literature after the story for exercise.

\section{Colour calculation}

153 All colours (colour shades) used for designing the slides (stimuli) were defined within the RGB

154 colour model and each individual colour was expressed as a RGB triplet $[\mathrm{R}, \mathrm{G}, \mathrm{B}]$, where the value 155 of each additive primary colour component can vary from 0 to 255 . List of background shades in 156 slides with coloured background (and black text) with associated numerical values of their RGB 157 triplet are: red ("red", [255,0,0]), blue ("blue", [0,0,255]), yellow ("yellow", [255,255,0]), orange

158 (“orange”, [255,128,0], purple (“purple”, [255,0,128]) and turquoise ("turquoise”, [0,255,255].

159 White and black shades were defined by triplets $[255,255,255]$ and $[0,0,0]$, respectively. RGB

160 components of the background and text in the slides with "overlay effect" were calculated

161 according to the following formula:

162 Overlay Component $=$ Shade Component $*$ Opacity $+(1-$ Opacity $) *$ Underlay Shade

163 where, Opacity value was set to 0.5 , Shade Component was selected from one of the previously

164 listed background colour shades, and Underlay Shade value in case of text being 0 for black and

165 in case of white background being 255.

166 The resulting RGB triplets for shades of the text and background for slides with overlay effect 167 were: overlay red ("red O", text - [128,0,0], background -[255,128,128]), overlay blue ("blue O", 168 text - $[0,0,128]$, background -[128,128,255]), overlay yellow ("yellow O", text - $[128,128,0]$, 
169 background -[255,255,128]), overlay orange (“orange O”, text - $[128,64,0]$, background -

$170[255,192,128])$, overlay purple (“purple O”, text - [128,0,64], background -[255,128,192]) and

171 overlay turquoise (“turquoise O", text - [0,128,128], background -[128,255,255]).

\section{Data processing}

173 Eye tracking data analysis and visualization was performed using BeGaze 3.7 software. The

174 selected eye-tracking parameters were: fixation count, fixation frequency (count/second), fixation

175 duration total (ms), fixation duration average (ms), saccade count, saccade frequency

176 (count/second), saccade duration total (ms), saccade duration average (ms).

177 EEG/ECG/EDA data were analysed using Matlab ver. 8.5 (Mathworks, USA) in the manner

178 described below.

179 For each subject and electrode site, EEG signal was processed in order to calculate the band power

180 in 5 predefined frequency bands: delta $(0.5-4 \mathrm{~Hz})$, theta $(4-7 \mathrm{~Hz})$, alpha $(7-13 \mathrm{~Hz})$, beta $(15$ -

$18140 \mathrm{~Hz})$ and whole range $(0.5-40 \mathrm{~Hz})$. Median value of EEG band power (for each frequency band)

182 was determined for 13-time epochs coinciding with reading of the content of each presented slide.

183 Median EEG power in each frequency band was calculated by raw continuous signal band-pass

184 filtering (4th order Butterworth filter with cut-off frequencies defined by individual band's

185 frequency range), squaring, segmenting into 13 epochs (determined as time intervals between each

186 slide's onset and offset) and median averaging to a single power value over signal samples of each

187 epoch (i.e. over each slide's duration). Applied processing resulted in the 65 median power values

188 (i.e. 13 slides x 5 frequency bands) for each EEG channel of each subject. Median power

189 calculation was applied since it is less likely to be affected by outliers in the EEG power samples

190 occurring with temporary movement artefacts than the mean power. 
191 Heart activity signal was band-pass filtered using a FIR filter in the range 1-45 Hz (750 points),

192 after which the Pan-Tompkins algorithm [21] for extraction of heart activity beats was applied.

193 Beat-to-beat intervals (BBI, the time between two successive heart activity peaks) were calculated.

194 Heart rate variability (HRV) parameters [22] were calculated from BBIs for 13-time epochs

195 coinciding with reading of the content of each presented slide, Table 1. Applied processing resulted

196 in the 14 HRV parameter values in one subject for each of the 13 slides.

197 Mean value of EDA data was calculated as a representative value of electrodermal activity for 13-

198 time epochs coinciding with reading of the content of each presented slide. Applied processing

199 resulted in the 13 mean values for each subject (one mean EDA value per slide).

Table1. HRV parameters

\begin{tabular}{|c|c|c|}
\hline Parameter & Unit & Description \\
\hline \multicolumn{3}{|l|}{ Time domain parameters } \\
\hline Mean RR & $\mathrm{ms}$ & Mean value of BBIs \\
\hline SDNN & $\mathrm{ms}$ & Standard deviation of normal BBIs \\
\hline Mean HR & beats/min & Mean value of heart rate \\
\hline STD HR & beats/min & Standard deviation of heart rate \\
\hline CVRR=SDNN/Mean RR & n.u. & Coefficient of variance of normal BBIs \\
\hline RMSSD & $\mathrm{ms}$ & Root mean square of differences of successive BBIs \\
\hline NN50 & beats & Number of successive BBIs that varied more than $50 \mathrm{~ms}$ \\
\hline pNN50 & $\%$ & Percentage of successive BBIs that differ more than $50 \mathrm{~ms}$ \\
\hline
\end{tabular}

\section{Statistical methodology}

205 Results are presented as count $(\%)$, means \pm standard deviation or depending on data type and 206 distribution. Groups are compared using parametric test, independent samples $\mathrm{t}$ test. All $\mathrm{p}$ values

207 less than 0.05 were considered significant. All data were analysed using SPSS 20.0 (IBM Corp.

208 Released 2011. IBM SPSS Statistics for Windows, Version 20.0. Armonk, NY: IBM Corp.) and

209 R 3.4.2. (R Core Team (2017). R: A language and environment for statistical computing. R

210 Foundation for Statistical Computing, Vienna, Austria. URL https://www.R-project.org/.). 


\section{Results}

213

214 White (default) background - reading results

215 Grade comparisons (second vs. third) regarding the examined parameters for white colour only are

216 presented in Table 2. A significant difference has been obtained regarding EEG frequency bands

217 (Alpha, Beta and Theta) and ECG parameters (SDNN, CVRR and STD HR). In all other

218 parameters we observed no significant difference between second and third graders. All EEG

219 power bands are higher in the second grade group (except Delta band), compared to third graders.

220 An opposite trend is shown in SDNN, CVRR and STD HR parameters whereby third graders

221 achieved higher scores in comparison to second graders.

224 Table 2. Reading duration, EEG, Eye tracking, EDA and HRV parameters in second and third 225 grade - significant $\mathrm{p}$ values are marked as bold

\begin{tabular}{|c|c|c|c|}
\hline \multirow{2}{*}{ Parameters } & \multicolumn{2}{|c|}{ Grade } & \multirow{2}{*}{$\mathrm{p}$ value } \\
\hline & Second $(n=12)$ & Third $(n=12)$ & \\
\hline \multicolumn{4}{|l|}{ Reading duration } \\
\hline $\mathrm{RD}(\mathrm{s})$ & $43.38 \pm 25.32$ & $49.07 \pm 27.63$ & 0.628 \\
\hline \multicolumn{4}{|c|}{ EEG parameters (median power band) } \\
\hline Alpha & $13.19 \pm 5.15$ & $5.63 \pm 4.28$ & 0.001 \\
\hline Beta & $6.05 \pm 2.81$ & $3.33 \pm 2.40$ & 0.018 \\
\hline Delta & $64.45 \pm 20.47$ & $71.85 \pm 63.54$ & 0.707 \\
\hline Theta & $16.07 \pm 7.14$ & $8.35 \pm 7.67$ & 0.018 \\
\hline Whole Range & $113.3 \pm 38.5$ & $97.2 \pm 80.5$ & 0.540 \\
\hline \multicolumn{4}{|l|}{ Eye tracking parameters } \\
\hline Fixation Count & $38.25 \pm 19.62$ & $35.00 \pm 9.66$ & 0.620 \\
\hline Fixation Frequency [count/s] & $1.01 \pm 0.36$ & $0.99 \pm 0.67$ & 0.925 \\
\hline Fixation Duration Total [s] & $40.87 \pm 25.01$ & $46.22 \pm 26.32$ & 0.631 \\
\hline Fixation Duration Average [ms] & $1088.2 \pm 542.9$ & $1331.5 \pm 730.9$ & 0.381 \\
\hline Saccade Count & $34.92 \pm 19.16$ & $28.70 \pm 5.23$ & 0.301 \\
\hline Saccade Frequency [count/s] & $0.95 \pm 0.38$ & $0.86 \pm 0.65$ & 0.686 \\
\hline Saccade Duration Total [ms] & $741.3 \pm 449.4$ & $680.0 \pm 276.7$ & 0.711 \\
\hline Saccade Duration Average [ms] & $20.93 \pm 2.76$ & $23.22 \pm 5.61$ & 0.227 \\
\hline \multicolumn{4}{|l|}{ EDA value } \\
\hline EDA (uS) & $9.41 \pm 3.49$ & $6.20 \pm 4.38$ & 0.060 \\
\hline \multicolumn{4}{|l|}{ HRV parameters } \\
\hline Mean RR (ms) & $637.4 \pm 59.8$ & $680.5 \pm 114.4$ & 0.264 \\
\hline SDNN (ms) & $35.14 \pm 11.95$ & $66.11 \pm 46.15$ & 0.043 \\
\hline CVRR (n.u.) & $0.06 \pm 0.02$ & $0.10 \pm 0.05$ & 0.021 \\
\hline Mean HR (beats/min) & $94.87 \pm 8.69$ & $90.26 \pm 13.75$ & 0.337 \\
\hline STD HR (beats/min) & $5.12 \pm 1.54$ & $8.40 \pm 3.85$ & 0.016 \\
\hline
\end{tabular}




\begin{tabular}{|l|c|c|c|}
\hline RMSSD (ms) & $42.50 \pm 17.42$ & $84.94 \pm 74.74$ & 0.079 \\
\hline NN50 (beats) & $12.42 \pm 9.44$ & $19.67 \pm 16.43$ & 0.298 \\
\hline pNN50 (\%) & $23.40 \pm 18.34$ & $36.30 \pm 25.88$ & 0.236 \\
\hline
\end{tabular}

\section{Background and overlay colours}

In the Table 2 we showed the overall results which were calculated by subtracting parameters acquired for white colour from parameters acquired for each of the background and overlay colours measured in the study, namely: Reading duration, EEG, Eye tracking, EDA and HRV.

233 The results demonstrated that students in the second grade differed significantly from the students

234 in the third grade consistently on a few HRV parameters, in particular: SDNN (yellow, turquoise

235 and turquoise O), CVRR (for orange, turquoise and blue O), and STD HR (blue, yellow, turquoise,

236 blue $\mathrm{O}$, yellow $\mathrm{O}$, orange $\mathrm{O}$ and turquoise $\mathrm{O}$ ). Besides these differences, significant differences

237 were found in RMSSD (for turquoise and blue O). In each of these cases second grade students 238 scored higher (or positively) in comparison to the third grade students who scored lower (or 239 negatively), which is marked with orange colour in Table 3. Out of all parameters in the study,

240 only in the case of Saccade Duration Total for the blue O, the third graders scored significantly

241 higher than the second graders, which is marked with green colour in Table 3.

Table 3. Differences between second and third graders on reading duration, EEG, Eye tracking, EDA and HRV parameters (normalized on white colour), $(p<.05$ is marked with "+", and $p<.01$ is marked with "++")

\begin{tabular}{|c|c|c|c|c|c|c|c|c|c|c|c|c|}
\hline \multirow{10}{*}{ Parameters } & \multicolumn{12}{|c|}{ Normalized values } \\
\hline & $r$ & b & $\mathrm{y}$ & 0 & $\mathrm{p}$ & $\mathrm{t}$ & r & b & $\mathrm{y}$ & o & $\mathrm{p}$ & $\mathrm{t}$ \\
\hline & e & l & e & $\mathrm{r}$ & $\mathrm{u}$ & $\mathrm{u}$ & $\mathrm{e}$ & l & $\mathrm{e}$ & $\mathrm{r}$ & $\mathrm{u}$ & $\mathrm{u}$ \\
\hline & $\mathrm{d}$ & $\mathrm{u}$ & l & a & $\mathrm{r}$ & $\mathrm{r}$ & $\mathrm{d}$ & $\mathrm{u}$ & l & $\mathrm{a}$ & $\mathrm{r}$ & $\mathrm{r}$ \\
\hline & & e & l & $\mathrm{n}$ & $\mathrm{p}$ & $\mathrm{q}$ & 0 & $\mathrm{e}$ & l & $\mathrm{n}$ & $\mathrm{p}$ & $\mathrm{q}$ \\
\hline & & & o & $\mathrm{g}$ & l & $\mathrm{u}$ & & 0 & 0 & $\mathrm{~g}$ & l & $\mathrm{u}$ \\
\hline & & & w & $\mathrm{e}$ & $\mathrm{e}$ & 0 & & & $\mathrm{w}$ & $\mathrm{e}$ & $\mathrm{e}$ & 0 \\
\hline & & & & & & $\mathrm{i}$ & & & 0 & 0 & 0 & $\mathrm{i}$ \\
\hline & & & & & & $\mathrm{s}$ & & & & & & $\mathrm{s}$ \\
\hline & & & & & & 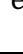 & & & & & & 0 \\
\hline
\end{tabular}




\begin{tabular}{|c|c|c|c|c|c|c|c|c|}
\hline \multicolumn{9}{|l|}{ Reading duration } \\
\hline $\mathrm{RD}(\mathrm{s})$ & & & & & & & & \\
\hline \multicolumn{9}{|l|}{$\begin{array}{l}\text { EEG parameters (median power } \\
\text { band) }\end{array}$} \\
\hline \multicolumn{9}{|l|}{ Alpha } \\
\hline \multicolumn{9}{|l|}{ Beta } \\
\hline \multicolumn{9}{|l|}{ Delta } \\
\hline \multicolumn{9}{|l|}{ Theta } \\
\hline \multicolumn{9}{|l|}{ Whole Range } \\
\hline \multicolumn{9}{|l|}{ Eye tracking parameters } \\
\hline \multicolumn{9}{|l|}{ Fixation Count } \\
\hline \multicolumn{9}{|l|}{ Fixation Frequency [count/s] } \\
\hline \multicolumn{9}{|l|}{ Fixation Duration Total [s] } \\
\hline \multicolumn{9}{|l|}{ Fixation Duration Average [ms] } \\
\hline \multicolumn{9}{|l|}{ Saccade Count } \\
\hline \multicolumn{9}{|l|}{ Saccade Frequency [count/s] } \\
\hline Saccade Duration Total [ms] & & & & & - & & & \\
\hline \multicolumn{9}{|l|}{ Saccade Duration Average [ms] } \\
\hline \multicolumn{9}{|l|}{ EDA value } \\
\hline \multicolumn{9}{|l|}{ EDA (uS) } \\
\hline \multicolumn{9}{|l|}{ HRV parameters } \\
\hline \multicolumn{9}{|l|}{ Mean RR (ms) } \\
\hline SDNN (ms) & & + & & ++ & & & & + \\
\hline CVRR & & & + & + & + & & & \\
\hline \multicolumn{9}{|l|}{ Mean HR (beats/min) } \\
\hline STD HR (beats/min) & + & + & & ++ & + & + & + & + \\
\hline RMSSD (ms) & & & & + & + & & & \\
\hline \multicolumn{9}{|l|}{ NN50 (beats) } \\
\hline pNN50 (\%) & & & & & & & & \\
\hline
\end{tabular}

In the Figure 2, normalized SDNN, CVRR and STD HR values across grades and colours are presented.

Figure 2. SDNN, CVRR and STD HR by grade and colour (normalized on white colour) Duration, Eye-tracking, EEG and EDA parameters, for a subsequent analysis all twelve background and overlay colours were averaged together in order to examine differences between younger and older children.

Grade comparisons (second vs. third) regarding the examined parameters over averaged scores for

Reading duration, median Alpha, Betha, Delta, Theta power bands as well as for the Whole range, 
261 Regarding Reading duration, third graders took significantly longer time to complete the reading.

262 All EEG power bands (Alpha, Betha, Delta, Theta and Whole range) as well as EDA were higher

263 in the second grade group, compared to third graders. An opposite trend was found for the eye-

264 tracking measures whereby both Fixation Duration Total and Fixation Duration Average were

265 higher in the third graders in comparison to the second graders. grade across all colours together - significant $p$ values are marked in bold

\begin{tabular}{|l|c|c|c|}
\hline \multirow{2}{*}{ Parameters } & \multicolumn{2}{|c|}{ Grade } & \multirow{2}{*}{ p value* } \\
\cline { 2 - 3 } & Second $(\mathrm{n}=156)$ & Third $(\mathrm{n}=156)$ & \\
\hline Reading duration & $42.74 \pm 31.81$ & $50.33 \pm 27.40$ & $\mathbf{0 . 0 0 1}$ \\
\hline RD $(\mathrm{s})$ & $13.50 \pm 5.28$ & $4.67 \pm 3.20$ & $\mathbf{0 . 0 0 1}$ \\
\hline EEG parameters (median power band) & $6.36 \pm 2.90$ & $3.71 \pm 4.70$ & $\mathbf{0 . 0 0 1}$ \\
\hline Alpha & $71.49 \pm 37.33$ & $51.23 \pm 48.90$ & $\mathbf{0 . 0 0 1}$ \\
\hline Beta & $17.55 \pm 7.88$ & $6.38 \pm 4.75$ & $\mathbf{0 . 0 0 1}$ \\
\hline Delta & $123.36 \pm 54.24$ & $74.45 \pm 61.30$ & $\mathbf{0 . 0 0 1}$ \\
\hline Theta & $35.14 \pm 13.97$ & $36.35 \pm 10.31$ & 0.412 \\
\hline Whole Range & $1.10 \pm 0.89$ & $1.01 \pm 0.75$ & 0.372 \\
\hline Eye tracking parameters & $40.01 \pm 30.71$ & $47.47 \pm 25.63$ & $\mathbf{0 . 0 2 8}$ \\
\hline Fixation Count & $1038.49 \pm$ & $1283.29 \pm$ & $\mathbf{0 . 0 0 1}$ \\
\hline Fixation Frequency [count/s] & 593.15 & 584.55 & \\
\hline Fixation Duration Total [s] & $31.31 \pm 12.36$ & $30.93 \pm 8.05$ & 0.753 \\
\hline Fixation Duration Average [ms] & $0.99 \pm 1.00$ & $0.89 \pm 0.71$ & 0.376 \\
\hline Saccade Count & $662.9 \pm 293.4$ & $712.0 \pm 273.6$ & 0.147 \\
\hline Saccade Frequency [count/s] & $21.65 \pm 6.09$ & $22.69 \pm 3.88$ & 0.093 \\
\hline Saccade Duration Total [ms] & $10.13 \pm 3.25$ & $6.32 \pm 3.95$ & $\mathbf{0 . 0 0 1}$ \\
\hline Saccade Duration Average [ms]
\end{tabular}

*independent sample $t$ test

\section{Discussion}

273 We have evaluated differences in Reading duration, EEG, eye tracking, EDA and HRV parameters

274 in 24 children (12 second and 12 third grade students of elementary school) using simultaneously

275 monitored sensor signals.

276 The literature regarding EEG power bands (Alpha, Beta, Delta, Theta) has been shown to have a

277 general developmental decreasing trend with an increasing age [23-25]. However, in the case of a 
278 specific mental activity, such as reading in this study, little is yet known about developmental 279 changes of contribution of EEG power bands [25]. When kids were given a task to read on a

280 white colour (which is a standard and everyday background colour), they showed a decrease in 281 Alpha, Beta and Theta power bands with age, Table 2. Given that this is a pilot study in this sense, 282 it remains to be resolved whether this is specifically a developmental trend, or one that has to do 283 with experience, or both in combination. To fully resolve this issue, one would also need to have 284 a much wider range of age groups tested.

285 Correlation between HRV parameters and age in infants and childhood, caused by progressive 286 maturation of the autonomous nervous system, is known in the literature [26-29]. However, the 287 age impact on HRV parameters is more extensive in infancy and the early childhood period (up to 288 eight years) [26]. In this study, participants were 8 and 9 years old, so they were in years of life 289 when the age impact was much less significant on HRV parameters [30]. Consequently, the level 290 of stress in our study was expected (and found) to be lower in the group with a higher 291 strain [31]. Namely, SDNN, CVRR and STD HR were found to be lower in younger children when 292 reading on white background, Table 2. However, we would not predominantly attribute these 293 findings to the developmental causal factor, but at least to some extent to experience in reading.

294 This study gives further support to existing findings that colours may play an important role in the 295 reading process $[13,15,16,18,32-36]$. When normalization on the white background was 296 performed (by subtracting white from each of the other background and overlay colours), 297 systematic differences (on at least two colours) were found regarding HRV parameters 298 (normalized SDNN, CVRR, and STD HR values), with second graders scoring higher on these 299 parameters, Table 3. The corresponding graphics across colours are presented in Figure 2, where 300 it could be observed that blue and turquoise backgrounds have a calming impact (increasing 
normalized SDNN, CVRR, and STD HR values) on second graders, which is in accordance with the previous reports [37-39]. No systematic differences were found across colours between

303 younger and older children in Reading duration, EEG power band, Eye-tracking and EDA

304 measures. This is why, based on the results presented in Table 3, for the following analyses all

305 twelve background and overlay colours were averaged together and examined differences between

306 second and third graders in that case, Table 4. Based on the results presented in Table 4, we

307 observed a significant difference across multimodal sensor measurements for reading on

308 background and overlay colours in contrast to reading on the white background colour.

310 The findings concerning Reading duration on background and coloured overlays showed that the

311 third-grade students have longer Reading duration in comparison to the second-graders. This could

312 be explained by the fact that third graders or older children need more time to adapt to unexpected

313 text and stimuli such as colour [40-42]. Also, previous studies have shown that older children are

314 slower in reading than younger children because they make longer fixation duration and saccades

315 [43-48] skipping words more frequently than younger [49,50]. Concerning the eye-tracking

316 measures, it was in fact found that third-graders have longer Fixation Duration Total and Fixation

317 Duration Average in comparison to the second-graders, which is a result that is in line with the

318 above mentioned assertion that older children take longer to read. This indicates higher mental

319 load in the reading task in older children.

320 A similar pattern of EEG results found for the white background was also found for the background

321 and overlay colours. However, the number of parameters which showed a significant difference

322 between second and third graders and significance levels were much more prominent for 323 background and overlay colours in comparison to the white background. The study shows 
324 significant differences between the second and third graders in all four EEG power bands (Alpha,

325 Beta, Delta and Theta), as well as in the Whole Range based on the averaged results from the

326 twelve background and overlay colours.

327 Similarly, it is important to mention that EDA linearly correlates to arousal and reflects cognitive

328 activity and emotional response [51,52] and it is the most used psychophysiological measure of

329 arousal [53]. The higher the arousal is, the higher electrodermal activity is. In the present study, it

330 was found to be higher among second graders, from which we conclude that they had a higher

331 stress-level.

332

333 Conclusion

334 The aim of this study was to assess developmental differences in second and third grade students

335 of elementary school regarding reading on white vs coloured overlay and background and to

336 evaluate differences in reading duration, brain, eye, electrodermal and heart activities. Evaluating

337 all findings and results during reading on white colour and all 12 background and overlay colours,

338 we can conclude that there is a decreasing trend with age regarding EEG power bands (Alpha,

339 Beta, Delta, Theta) that is shown in the comparison between second and third grade students. In

340 addition, second graders show lower scores of reading duration and eye-tracking measures

341 (fixation duration total and fixation duration average), which confirms the fact that older children

342 need more time to adapt on unexpected text and have longer fixations on words during reading.

343 Comparing HRV parameters in second and third graders during reading on white colour, we have

344 found lower scores in second graders and higher scores in 12 overlays and background colours

345 compared to third graders, especially for SDNN, CVRR, and STD HR measures. The highest

346 values of normalized SDNN, CVRR, and STD HR among students were reached in turquoise and 
347 blue background colours, which could be the result of calming effect during task performance 348 caused by the background colour. Furthermore, we have found that EDA linearly correlates to

349 level of arousal (tension and stress), where we have found higher values in younger children.

350 Across single colours and its influence on measures during the reading task, there is no systematic

351 difference in Reading duration, EEG power band, Eye-tracking and EDA measures, except for

352 HRV measures as mentioned above.

353 Thus, the goal of combining different modalities was to find a more objective approach to

354 understanding the developmental differences in children's reading as well as to understand the

355 contribution of different modalities and combinations of modalities in the process of reading text

356 on a white vs colour overlay and background.

357 In the following work, it will be necessary to move forward from group studies to individual 358 studies in order to determine and establish individual optimal parameters, as well as colours 359 corresponding to individual differences in the reading process.

\section{Acknowledgment}

362 The authors acknowledge the financial support from the Slovenian Research Agency (research 363 core funding No. P2-0098), AD Futura Found (Public Scholarship, Development, Disability and

364 Maintenance Found of the Republic of Slovenia), IPS Jozef Stefan and the Ministry of Education, 365 Science and Technological Development of the Republic of Serbia.

\section{References}

369 1. Schroeder S, Hyönä J, Liversedge SP. Developmental eye-tracking research in reading: Introduction to the special issue [Internet]. Vol. 27, Journal of Cognitive Psychology. Psychology Press Ltd; 2015 
371

372

373

374

375

376

377

378

379

380

381

382

383

384

385

386

387

388

389

390

391

392

393

394

395

396

397

398

399

400

401

402

403

404

405

406

407

408

409

410

411

412

413

414

415

416

417

418

419

420

[cited 2020

Jul

11].

p.

500-10.

Available

from:https://www.tandfonline.com/doi/abs/10.1080/20445911.2015.1046877

2. Korneev AA, Matveeva EY, Akhutina T v. What We Can Learn about Reading Development from the Analysis of Eye Movements. Human Physiology. 2018 Mar 1;44(2):183-90.

3. Lobier M, Dubois M, Valdois S. The Role of Visual Processing Speed in Reading Speed Development. Barton JJS, editor. PLoS ONE [Internet]. 2013 Apr 4 [cited 2020 Mar 30];8(4):e58097. Available from: http://dx.plos.org/10.1371/journal.pone.0058097

4. Lerkkanen MK, Rasku-Puttonen H, Aunola K, Nurmi JE. Reading performance and its developmental trajectories during the first and the second grade. Learning and Instruction. $2004 \mathrm{Apr}$ $1 ; 14(2): 111-30$.

5. Miller B, Shriver EK, O'donnell C. Opening a Window into Reading Development: Eye Movements' Role Within a Broader Literacy Research Framework. Vol. 42, School Psych Rev. 2013.

6. Korneev AA, Akhutina T v, Matveeva EYu. Reading in third graders with different state of the skill: an eye-tracking study. Moscow University Psychology Bulletin. 2019;2:64-87.

7. Vorstius C, Radach R, Lonigan CJ. Eye movements in developing readers: A comparison of silent and oral sentence reading. Visual Cognition. 2014;22(3):458-85.

8. Hulme C, Snowling MJ. Learning to Read: What We Know and What We Need to Understand Better.

9. McArthur G, Castles A. Helping children with reading difficulties: some things we have learned so far. npj Science of Learning [Internet]. 2017 Dec 31 [cited 2020 Jul 11];2(1):7. Available from:www.motif.org.au

10. van Bommel WJM, van den Beld GJ. Lighting for work: A review of visual and biological effects. Lighting Research and Technology [Internet]. 2004 Dec 19 [cited 2020 Mar 4];36(4):255-69. Available from: http://journals.sagepub.com/doi/10.1191/1365782804li122oa

11. de Jong PF, van der Leij A. Effects of Phonological Abilities and Linguistic Comprehension on the Development of Reading. Scientific Studies of Reading. 2002 Jan;6(1):51-77.

12. Uccula A, Enna M, Mulatti C. Colors, colored overlays, and reading skills. Frontiers in Psychology [Internet]. $2014 \quad$ Jul 29 [cited 2020 Jul 10];5(JUL):833. Available from:http://journal.frontiersin.org/article/10.3389/fpsyg.2014.00833/abstract

13. Conway ML, Evans BJW, Evans JC, Suttle CM, Engel FL, Child PBASIC, et al. Colors, colored overlays, and reading skills. Allen P, editor. Frontiers in Psychology [Internet]. 2014 Sep 20 [cited 2019 Jun 10];5(7):9-21. Available from: www.frontiersin.org

14. Jansky JJ. A case of severe dyslexia with aphasic-like symptoms. Bulletin of the Orton Society [Internet]. 1958 May [cited 2020 May 5];8(1):8-11. Available from:http://link.springer.com/10.1007/BF02657600

15. Wilkins AJ, Evans BJW. Visual stress, its treatment with spectral filters, and its relationship to visually induced motion sickness. Applied Ergonomics [Internet]. 2010 Jul [cited 2020 Mar 3];41(4):509-15. Available from:https://linkinghub.elsevier.com/retrieve/pii/S0003687009000325

16. Pinna B, Deiana K. On the Role of Color in Reading and Comprehension Tasks in Dyslexic Children and Adults. i-Perception [Internet]. 2018 May 9 [cited 2020 Apr 10];9(3):204166951877909. Available from:http://journals.sagepub.com/doi/full/10.1177/2041669518779098

17. Denton TF, Meindl JN. The Effect of Colored Overlays on Reading Fluency in Individuals with Dyslexia. Behavior Analysis in Practice [Internet]. 2016 Sep [cited 2020 Jul 10];9(3):191-8. Available from: /pmc/articles/PMC4999357/?report=abstract

18. Veszeli J, Shepherd AJ. A comparison of the effects of the colour and size of coloured overlays on young children's reading. Vision Research. 2019 Mar 1;156:73-83.

19. Sam W. The use of eye tracking with infants and children. Practical Research With Children. Routledge. 2016; pp.24-45.

20. Giagloglou E, Radenkovic M, Brankovic S, Antoniou P, Zivanovic-Macuzic I. Pushing, pulling and manoeuvring an industrial cart: a psychophysiological study. International Journal of 
Occupational Safety and Ergonomics [Internet]. 2019 Apr 3 [cited 2020 Jul 13];25(2):296-304. Available from: https://pubmed.ncbi.nlm.nih.gov/28849989/

21. Sachin S, Netaji G N. Pattern analysis of different ECG signal using PanTompkin's algorithm. (IJCSE) International Journal on Computer Science and Engineering. 2010.

22. Shaffer F, Ginsberg JP. An Overview of Heart Rate Variability Metrics and Norms. Frontiers in Public Health [Internet]. 2017 Sep 28 [cited 2020 Jul 11];5:258. Available from: /pmc/articles/PMC5624990/?report=abstract

23. Marshall PJ, Bar-Haim Y, Fox NA. Development of the EEG from 5 months to 4 years of age. Clinical Neurophysiology. 2002 Aug 1;113(8):1199-208.

24. Miskovic V, Ma X, Chou C-A, Fan M, Owens M, Sayama H, et al. Developmental changes in spontaneous electrocortical activity and network organization from early to late childhood.NeuroImage [Internet]. 2015 Sep 1 [cited 2020 May 24];118:237-47. Available from:https://linkinghub.elsevier.com/retrieve/pii/S1053811915005108

25. Spironelli C, Angrilli A. Developmental aspects of language lateralization in delta, theta, alpha and beta EEG bands. Biological Psychology [Internet]. 2010 Oct [cited 2020 Jul 12];85(2):258-67. Available from: https://pubmed.ncbi.nlm.nih.gov/20659528/

26. Massin M, von Bernuth G. Normal ranges of heart rate variability during infancy and childhood. Pediatric Cardiology. 1997 Jul;18(4):297-302.

27. Goto M, Nagashima M, Baba R, Nagano Y, Yokota M, Nishibata K, et al. Analysis of heart rate variability demonstrates effects of development on vagal modulation of heart rate in healthy children. Journal of Pediatrics. 1997;130(5):725-9.

28. Finley JP, Nugent ST. Heart rate variability in infants, children and young adults. Journal of the Autonomic Nervous System [Internet]. 1995 Feb 9 [cited 2020 May 25];51(2):103-8. Available from: https://linkinghub.elsevier.com/retrieve/pii/0165183894001173

29. Seppälä S, Laitinen T, Tarvainen MP, Tompuri T, Veijalainen A, Savonen K, et al. Normal values for heart rate variability parameters in children 6-8 years of age: the PANIC Study. Clinical Physiology and Functional Imaging [Internet]. 2014 Jul [cited 2020 May 25];34(4):290-6. Available from: http://doi.wiley.com/10.1111/cpf.12096

30. Kim HG, Cheon EJ, Bai DS, Lee YH, Koo BH. Stress and heart rate variability: A meta-analysis and review of the literature [Internet]. Vol. 15, Psychiatry Investigation. Korean Neuropsychiatric Association; 2018 [cited 2020 Jul 12]. p. 235-45. Available from: /pmc/articles/PMC5900369/?report=abstract

31. Kang MG, Koh SB, Cha BS, Park JK, Woo JM, Chang SJ. Association between job stress on heart rate variability and metabolic syndrome in shipyard male workers. Yonsei Medical Journal. 2004 Oct 31;45(5):838-46.

32. Freiders S, Lee S, Statz D, Kim Group T. The Influence of Color on Physiological Response.

33. Morrison R. Effect of Color Overlays on Reading Efficiency [Internet]. 2011 [cited 2020 Apr 10]. Available from: https://scholarworks.umass.edu/open access dissertations/431

34. Griffiths PG, Taylor RH, Henderson LM, Barrett BT. The effect of coloured overlays and lenses on reading: a systematic review of the literature. Ophthalmic and Physiological Optics [Internet]. 2016 Sep 1 [cited 2020 Apr 10];36(5):519-44. Available from:http://doi.wiley.com/10.1111/opo.12316

35. Rello L, Bigham JP. Good Background Colors for Readers: A Study of People with and without Dyslexia. 2017 [cited 2020 Apr 10]; Available from: https://doi.org/10.1145/3132525.3132546

36. Hlengwa N, Moonsamy P, Ngwane F, Nirghin U, Singh S. The effect of color overlays on the reading ability of dyslexic children [Internet]. Vol. 65, Indian Journal of Ophthalmology.Medknow Publications; 2017 [cited 2020 Jul 12]. p. 772-3. Available from:https://www.ncbi.nlm.nih.gov/pmc/articles/PMC5598199/ 
37. AL-Ayash A, Kane RT, Smith D, Green-Armytage P. The influence of color on student emotion, heart rate, and performance in learning environments. Color Research \& Application [Internet]. 2016 Apr 1 [cited 2020 May 24];41(2):196-205. Available from:http://doi.wiley.com/10.1002/col.21949

38. Mehta R, Zhu R. Blue or red? Exploring the effect of color on cognitive task performances. Science [Internet]. 2009 Feb 27 [cited 2020 Mar 4];323(5918):1226-9. Available from:https://www.sciencemag.org/lookup/doi/10.1126/science.1169144

39. Moharreri S., Dabanloo N. J., Parvaneh S., \& Nasrabadi A. M. How to interpret psychology from heart rate variability? 2011 1st Middle East Conference on Biomedical Engineering. 2011. doi:10.1109/mecbme.2011.5752124

40. Rayner K, Liversedge SP, White SJ. Eye movements when reading disappearing text: The importance of the word to the right of fixation. Vision Research. 2006 Feb 1;46(3):310-23.

41. Rayner K, Yang J, Schuett S, Slattery TJ. Eye movements of older and younger readers when reading unspaced text. Experimental psychology. 2013;60(5):354-61.

42. Rayner K, Castelhano MS, Yang J. Eye Movements and the Perceptual Span in Older and Younger Readers. Psychology and Aging. 2009.

43. Kliegl R, Grabner E, Rolfs M, Engbert R. Length, frequency, and predictability effects of words on eye movements in reading. In: European Journal of Cognitive Psychology. 2004. p. 262-84.

44. Dambacher M, Kliegl R, Hofmann M, Jacobs AM. Frequency and predictability effects on event-related potentials during reading. Brain Research. 2006;1084(1):89-103.

45. Rayner K, Castelhano MS, Yang J. Preview benefit during eye fixations in reading for older and younger readers. Psychology and Aging. 2010;25(3):714-8.

46. Rayner K, Chace KH, Slattery TJ, Ashby J. Eye movements as reflections of comprehension processes in reading. Vol. 10, Scientific Studies of Reading. 2006;p. 241-55.

47. Rayner K. Eye movements and the perceptual span in beginning and skilled readers. Journal of Experimental Child Psychology. 1986;41(2):211-36.

48. Rayner K, Foorman BR, Perfetti CA, Pesetsky D, Seidenberg MS. How Psychological Science Informs the Teaching of Reading. Psychological Science in the Public Interest. 2001;2(2):31-74.

49. Laubrock J, Klieg1 R, Engbert R. SWIFT explorations of age differences in eye movements during reading. Vol. 30, Neuroscience and Biobehavioral Reviews. 2006; p. 872-84.

50. Rayner K, Reichle ED, Stroud MJ, Williams CC, Pollatsek A. The effect of word frequency, word predictability, and font difficulty on the eye movements of young and older readers. Psychology and Aging. 2006;21(3):448-65.

51. Boucsein Wolfram. Electrodermal activity. Springer Science+Business Media, LLC. 2012; p. 618.

52. Boucsein W, Boucsein W. Principles of Electrodermal Phenomena. In: Electrodermal Activity. Springer US; 2012; p. 1-86.

53. Carlucci L, Watkins MW, Sergi MR, Cataldi F, Saggino A, Balsamo M. Dimensions of anxiety, age, and gender: Assessing dimensionality and measurement invariance of the State-Trait for Cognitive and Somatic Anxiety (STICSA) in an Italian sample. Frontiers in Psychology. 2018 ;p. 2345. 
